\title{
ON THE CONVERGENCE OF TRIGONOMETRIC APPROXIMATIONS FOR A FUNCTION OF TWO VARIABLES*
}

\section{BY ELIZABETH CARLSON}

A discussion of the convergence of approximating functions for a given function of two variables $f(x, y)$, just as in the case of functions of one variable, can be based on two sets of theorems: (1) theorems on the existence of functions of closest approximation; (2) theorems on the representation of $f(x, y)$ by means of finite sums constructed in a specific way.

From the first group we shall make use of the following theorem:

Theorem I. Let $p_{1}(x, y), p_{2}(x y), \cdots, p_{N}(x, y)$ be $N$ functions of $x$ and $y$, continuous in the region $R$ : ( $a \leqq x \leqq b$, $c \leqq y \leqq d)$, and linearly independent in this region. Let

$$
\phi(x, y)=c_{1} p_{1}(x, y)+c_{2} p_{2}(x, y)+\cdots+c_{N} p_{N}(x, y)
$$

be an arbitrary linear combination of the given functions with constant coefficients. Let $f(x, y)$ be a continuous function of $x$ and $y$ in $R$. Then there exists a choice of the coefficients $c_{k}$ in $\phi(x, y)$ such that the integral

$$
\int_{a}^{b} d x \int_{c}^{d}|f(x, y)-\phi(x, y)|^{m} d y, \quad(m>0),
$$

has its minimum value. The function $\phi(x, y)$ so determined is unique for $m>1$. It is called an approximating function for $f(x, y)$ corresponding to the exponent $m$.

This theorem can be proved by methods analogous to those used in proving the corresponding theorem for functions of a single variable. $\dagger$ In this paper we shall choose

* Presented to the Society, April 14, 1922.

$\dagger$ Jackson, On functions of closest approximation, Transactions of THIS Society, vol. 22 (1921), pp. 117-128; Note on an ambiguous case of approximation, Transactions, vol. 25 (1923), pp. 333-337. 
the $p$ 's so that a function of the form $\phi(x, y)$ with arbitrary coefficients is an arbitrary trigonometric sum of order $n$ in $x$ and of the same order in $y$, and we shall take as the region $R$ the square $0 \leqq x \leqq 2 \pi, 0 \leqq y \leqq 2 \pi$.

From the second group of theorems, we shall apply the following theorems.

THEOREM II. If $f(x, y)$, of period $2 \pi$ in each argument, has a modulus of continuity $\omega(\delta)$, then $f(x, y)$ can be represented everywhere by a trigonometric sum $t_{n}(x, y)$, of order $n$ in each variable, with an error that does not exceed a constant times $\omega(2 \pi / n)$.

THEOREM III. If $f(x, y)$ has continuous first partial derivatives, it can be approximately represented with a maximum error $\epsilon_{n}$ such that $\lim _{n \rightarrow \infty} n \epsilon_{n}=0$.

The assertion II can be proved once more by an adaptation of the reasoning used in the case of functions of a single variable.* A proof of III was communicated to the writer orally by Professor Jackson.

Theorems I and II lead to the following theorem for the convergence of $T_{n}(x, y)$, the trigonometric approximating function, of order $n$ in each variable, corresponding to the exponent $m(m>1)$.

THEOREM IV. If $f(x, y)$, of period $2 \pi$ in each argument, is everywhere continuous, with a modulus of continuity $\omega(\delta)$, then a sufficient condition for the uniform convergence of $T_{n}(x, y)$ to $f(x, y)$ is $\dagger$

$$
\lim _{\delta \rightarrow 0} \omega(\delta) / \delta^{2 / m}=0 .
$$

* Jackson, On approximation by trigonometric sums and polynomials, Transactions of this Society, vol. 13 (1912), pp. 491-515. Cf. C. E. Wilder, On the degree of approximation to discontinuous functions by trigonometric sums, RENDICONTI DI PALERMO, vol. 39 (1915), pp. 345-361.

$\dagger \mathrm{Cf}$. Jackson, On the convergence of certain trigonometric and polynomial approximations, Transactions of THIS Society, vol. 22 (1921), pp. 158-166. 
The occurrence of the exponent $2 / m$, where $1 / m$ is found in the case of functions of a single variable, is due to the fact that the magnitude of an interval of length $1 / n$ is replaced in the course of the present reasoning by the area of a square having a quantity of the order of $1 / n$ for the length of its side.

This result is significant only if $m>2$, since otherwise the condition as stated requires that $f(x, y)$ be constant. When $m=2$, it is sufficient (in consequence of III) that $f(x, y)$ have continuous first partial derivatives. There are corresponding results for $m<2$.

The reasoning is not materially changed if the $m$ th power in the integral to be minimized is multiplied by a positive measurable weight function $\rho(x, y)$ with a positive lower bound.

The University of Minnesota

\section{ON A TENSOR OF THE SECOND RANK IN FUNCTION SPACE*}

\section{BY DUNHAM JACKSON}

In a recent paper $\dagger$ the writer has discussed a doubly infinite matrix of derivatives which has the properties of a tensor of the second rank in function space, and a quantity obtained by contraction of this tensor, which is analogous to a divergence. The latter concept is suggested formally by the writing of an infinite series, the general term of which in many cases does not approach zero; but an example was constructed in which the series is convergent, and defines a quantity which can be alternatively expressed in a form independent of any particular coordinate system.

* Presented to the Society, September 9, 1926.

$\dagger \mathrm{D}$. Jackson, Some convergence proofs in the vector analysis of function space, Annals of Mathematics, vol. 27, pp. 551-567. 